\title{
Optimal Control Problem of Treatment for Obesity in a Closed Population
}

\author{
D. Aldila, N. Rarasati, N. Nuraini, and E. Soewono \\ Department of Mathematics, Faculty of Mathematics and Natural Sciences, Institut Teknologi Bandung, \\ P.O. Box 40132, Bandung, Indonesia
}

Correspondence should be addressed to D. Aldila; aldiladipo@yahoo.co.id

Received 12 October 2013; Revised 12 January 2014; Accepted 16 January 2014; Published 26 February 2014

Academic Editor: Tamer Eren

Copyright (C) 2014 D. Aldila et al. This is an open access article distributed under the Creative Commons Attribution License, which permits unrestricted use, distribution, and reproduction in any medium, provided the original work is properly cited.

\begin{abstract}
Variety of intervention programs for controlling the obesity epidemic has been done worldwide. However, it is still not yet available a scientific tool to measure the effectiveness of those programs. This is due to the difficulty in parameterizing the human interaction and transition process of obesity. A dynamical model for simulating the interaction between healthy people, overweight people, and obese people in a randomly mixed population is discussed in here. Two scenarios of intervention programs were implemented in the model, dietary program for overweight people with healthy life campaign and treatment program for obese people. Assuming all control rates are constant, disease free equilibrium point, endemic equilibrium point, and basic reproductive ratio $\left(\mathscr{R}_{0}\right)$ as the epidemic indicator were shown analytically. We find that the disease free equilibrium point is locally asymptotical stable if and only if $\mathscr{R}_{0}<1$. From sensitivity analysis of $\mathscr{R}_{0}$, we obtain that larger rate of dietary program and treatment program will reduce $\mathscr{R}_{0}$ significantly. With control rates are continuous in time, an optimal control approach was applied into the model to find the best way to minimize the number of overweight and obese people. Some numerical analysis and simulations for optimal control of the intervention were shown to support the analytical results.
\end{abstract}

\section{Introduction}

Obesity is an overweight situation in human body as a result of excessive accumulation of fat situation. Every person needs some calories to save them energy, as well as to keep their body warm, and for many other purpose. The high consumption of high calorc food, over nutrition, and fast food combining with less physical activity to burn the calories become the main factors that cause obesity. The normal comparison between body fat with peoples weight is $18-23$ percent for men and 25-30 percents for women [1].

Obesity has reached the epidemic proportions since recent decades [2]. It became a worldwide problem as stated in the WHO report [3]; there are more than 1 billion overweight adults and at least 300 million of them obese. Obesity could lead to chronic diseases, including diabetes type 2, cardiovascular disease, hypertension, stroke, and certain forms of cancer [3].

Several factors have been identified in determining the susceptibility for obesity such as human genes and also energy balance. Calory intake and also physical activity are the main factors in energy balance.

Many programs have been initialized by WHO to solve the obesity pandemic such as recognizing the heavy criteria and growing burden of noncommunicable disease and developing global strategy on diet, physical activity, and health through broad consultation processes $[1,4]$. Promoting healthy behaviours to encourage, motivate, and enable individuals to lose weight by eating more fruit and vegetables, doing more physical activity, and reducing the consumption of sugars food have been done around the world recently. The problem is how to calculate the effectiviveness of these programs in long time period.

Some mathematical models have been developed to understand this disease like in $[5,6]$. Authors in [5] modeled that the obesity could spread by social interaction among people with statistical approach. SI deterministic mathematical model has been developed by the authors in [6]. Based on longitudinal data, the authors extract and compare the rates of spontaneous versus contagious acquisition of a behavior 
to predict the implications for future prevalence and control strategies.

Unlike the authors in $[5,6]$, the mathematical model of obese disease with vertical transmission will be constructed in this paper. Intervention programs such as healthy life campaign and rehabilitation program are applied in the model as control variable which depends on time. Mathematical assumption and the construction of the model will be shown in the next section. Mathematical analysis for equilibrium points and basic reproductive ratio is shown in Section 2. Optimal control problem and some numerical results are given in Sections 3 and 4, respectively. Some conclusions are given in the last section.

\section{Formulation of the Model}

Mathematical model for obesity in a closed population will be constructed in this section. We assume that the human population is divided into three different compartments, that is, healthy compartment $(x)$, overweight compartment $(y)$, and obese compartment $(z)$. The human population, denoted by $K$, is assumed to be constant with the per capita mortality rate given by $\mu$. We also assumed that obesity does not cause death in human population.

According to $[7,8]$, obese disease could be transmitted vertically to new born population through genetics. We assume that each compartment has the same recruitment rate for new borns, denoted by $\theta$. New borns from healthy people are 100 percent healthy, while if the parents are overweight or obese, then a portion of newborns will be healthy or overweight denoted by $p$ and $q$, respectively.

Transitions from healthy to overweight compartment depends on daily social interaction between healthy people with overweight and/or obese people with interaction coefficient given by $b$. With the same assumption, transition from overweight to obese compartment also depends on daily social interaction with obese people with interaction coefficient given by $b$ and also transition due to unhealthy living habits with rate of $\alpha$.

We assume that recovery rate from overweight compartment to healthy compartment is $\gamma$, while transition rate from obese compartment to overweight compartment could not happen naturally. Intervention programs such as healthy life style campaign is applied in the model to increase transition rate from overweight compartment to healthy compartment with rate of $u_{1}$. Treatment intervention programme is given to cure obese people with the rate of $u_{2}$.

From the assumptions above which is illustrated by the transmission diagram given in Figure 1 and parameter description in Table 1, we introduce the dynamical system

$$
\begin{aligned}
\frac{d x}{d t}= & \theta(x+p y+q z)+\left(\gamma+u_{1}(t)\right) y \\
& -\left(\frac{b}{K} y+\frac{b}{K} z\right) x-\mu x
\end{aligned}
$$

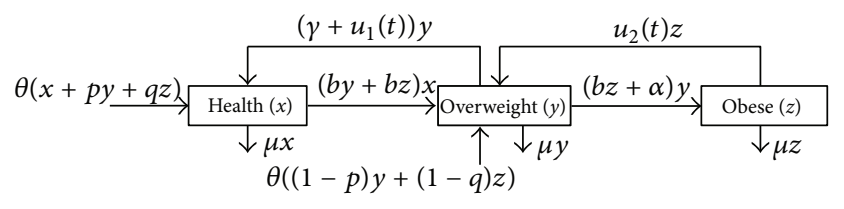

FIGURE 1: Transmission diagram for obese disease.

$$
\begin{aligned}
\frac{d y}{d t}= & \theta((1-p) y+(1-q) z) \\
& +\left(\frac{b}{K} y+\frac{b}{K} z\right) x+u_{2}(t) z \cdots \\
& -\left(\gamma+u_{1}(t)\right) y-\left(\frac{b}{K} z+\alpha\right) y-\mu y \\
\frac{d z}{d t} & =\left(\frac{b}{K} z+\alpha\right) y-u_{2}(t) z-\mu z
\end{aligned}
$$

The parameters of $u_{1}$ and $u_{2}$ applied in the model as the control variables depend on time. This should be done to accomodate the limitation of budget for campaign and rehabilitation program. For mathematical analysis of the equilibrium points and construction of the basic reproductive ratio which will be discussed in the next section, we first consider that these control variables $u_{1}(t)$ and $u_{2}(t)$ are constant.

\section{Analysis of the Model}

3.1. Equilibrium Points. In order to obtain the equilibrium points of system (1), we assume all control variables are constant and given by $u_{1}$ and $u_{2}$. We also assume that the recruitment rate for new borns is same with natural mortality to avoid the blow-up disappearance of all compartments $(\theta=$ $\mu$ ). Taking right hand side of (1) to be equal to 0 , the system (1) will have two different equilibrium points, that is, diseasefree equilibrium (DFE) where all infected population is equal to 0 and endemic equilibrium (EE) where all compartments exist in the field.

The disease-free equilibrium point is given by

$$
\mathrm{DFE}=\left(x^{*}, y^{*}, z^{*}\right)=(K, 0,0),
$$

where $K$ is the total population. The endemic equilibrium point is given by

$$
\begin{aligned}
\mathrm{EE} & =(\widehat{x}, \widehat{y}, \widehat{z}) \\
& =\left(\frac{g}{\left(u_{2} K+\mu K+b \widehat{z}+\alpha K\right) b}, \frac{\widehat{z} K\left(\mu+u_{2}\right)}{b \widehat{z}+\alpha K}, \widehat{z}\right),
\end{aligned}
$$

where $g=K\left(p \mu K u_{2}+p \mu^{2} K+\mu q b \widehat{z}+\mu q \alpha K+K \gamma u_{2}+K \gamma \mu+\right.$ $\left.K u_{1} u_{2}+K u_{1} \mu\right)$ and satisfy $\widehat{x}+\widehat{y}+\widehat{z}=K$. 
TABLE 1: Parameters description and value.

\begin{tabular}{|c|c|c|}
\hline Par. & Description & Value \\
\hline$\theta$ & Natural recruitment rate (per day) & $1 /(65 \times 365)$ \\
\hline$\mu$ & Natural mortality rate (per day) & $1 /(65 \times 365)$ \\
\hline$b$ & Interaction coefficient (per day) & $0.1 / K$ \\
\hline$\alpha$ & $\begin{array}{l}\text { Infection rate because bad life habit (per } \\
\text { day) }\end{array}$ & 0.05 \\
\hline$\gamma$ & $\begin{array}{l}\text { Natural recovery rate from overweight to } \\
\text { healthy comp. (per day) }\end{array}$ & 0.05 \\
\hline K & Total of human population (individuals) & 1000 \\
\hline$p$ & $\begin{array}{l}\text { Portion of health recruitment rate from } \\
\text { overweight comp. }\end{array}$ & 0.5 \\
\hline$q$ & $\begin{array}{l}\text { Portion of health recruitment rate from } \\
\text { obese comp. }\end{array}$ & 0.5 \\
\hline$u_{1}$ & Health life campaign rate (per day) & {$[0,1]$} \\
\hline$u_{2}$ & Treatment rate for obese people (per day) & {$[0,1]$} \\
\hline
\end{tabular}

3.2. Basic Reproductive Ratio. Using the next generation matrix operator approach in [9], we compute the basic reproductive ratio $\mathscr{R}_{0}$ which is associated with the diseasefree equilibrium (DFE). The basic reproductive ratio $\mathscr{R}_{0}$ represents the expected numbers of secondary cases produced by a typical infected individual during the entire period of infectiousness in a virgin population [10]. First of all, to construct the next generation matrix, we define

$$
\dot{A}=(G+T) A,
$$

where $A=(y, z)^{\text {transpose }}, G$ is transition matrix, and $T$ is transmission matrix. Transition matrix describes change in state, including removal rate like death or immunity, while transmission rate describes the production of new infections. The transition matrix $G$ from the infected compartment (overweight and obese) is given by

$$
G=\left[\begin{array}{cc}
(1-p) \mu-\alpha-\gamma-u_{1}-\mu & (1-q) \mu+u_{2} \\
\alpha & -u_{2}-\mu
\end{array}\right],
$$

while the transmission matrix $T$ is given by

$$
T=\left[\begin{array}{ll}
b & b \\
0 & 0
\end{array}\right]
$$

because in disease free equilibrium the whole population is healthy which is equal to $K$. In $[9,11]$, the basic reproductive ratio is equal to spectral radius of the matrix $T G^{-1}$. It can be seen that the matrix $T G^{-1}$ which is called the next generation matrix is given by

\section{NGM}

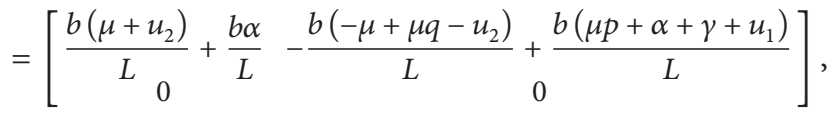

where $L=p \mu u_{2}+\mu^{2} p+\alpha \mu q+\gamma u_{2}+\mu \gamma+u_{1} u_{2}+\mu u_{1}$. Further detail about construction of next generation matrix can be seen in [9]. Recall that the spectral radius of a matrix is equal to its largest eigen value; therefore, the basic reproductive ratio is given by

$$
\mathscr{R}_{0}=\frac{b\left(\mu+u_{2}+\alpha\right)}{\mu^{2} p+\left(p u_{2}+q \alpha+\gamma+u_{1}\right) \mu+u_{2}\left(\gamma+u_{1}\right)} .
$$

We find that the disease-free equilibrium will be locally asymptotically stable if and only if $\mathscr{R}_{0}<1$.

Figure 2 shows the sensitivity between some parameters. Figure 2(a) shows the level set of $\mathscr{R}_{0}$ with respect to the value of healthy life campaign $u_{1}$ and treatment intervention $u_{2}$. It can be seen that the larger the number of both these interventions, then the value of basic reproductive ratio $\mathscr{R}_{0}$ will also decrease significantly. Unfortunately, larger rate of $u_{1}$ and $u_{2}$ needs more cost for implementation. Therefore, we have to find an optimal way to implement these intervention such that we can minimize number of overweight people and obese people with cost as low as possible.

Figure 2(b) is given to show the sensitivity between infection rate because of bad life habit $(\alpha)$ and natural recovery rate $(\gamma)$. It can be seen that with constant value of natural recovery rate, the value of basic reproductive ratio is increasing when transitioning to obese compartment because bad life habit also increase. Moreover, with constant value of transition because bad life habit rate from overweight to obese compartment, the basic reproductive ratio will decrease when natural recovery rate increasing.

In the next section, characterization of optimal control problem to reduce the number of overweight and obese compartments with intervention of health life campaign and treatment intervention will be given.

\section{Optimal Control Problem Characterization}

Our purpose in this model is to minimize number of overweight and obese compartments and the corresponding control functions. Together with the mathematical model of obese disease in (1), we consider an optimal control problem with the objective functional given by

$$
\begin{aligned}
& J\left(u_{i}, \Omega\right) \\
& \quad=\int_{0}^{T}\left(\omega_{x} x^{2}+\omega_{y} y^{2}+\omega_{z} z^{2}+\omega_{u_{1}} u_{1}^{2}+\omega_{u_{2}} u_{2}^{2}\right) d t,
\end{aligned}
$$

where $\Omega$ is the set of all compartment variables. $\omega_{x}, \omega_{y}$, and $\omega_{z}$ are weight constants for healthy compartment, overweight compartment, and obese compartment, respectively. Here we want to minimize the number of infected population; therefore, we set $\omega_{y}>0$ and $\omega_{z}>0$, while $\omega_{x}=0$. The weigth parameters $\omega_{u_{1}}$ and $\omega_{u_{2}}$ are weight constants for health life campaign $\left(u_{1}(t)\right)$ and treatment intervention $\left(u_{2}(t)\right)$ which regularize the optimal control condition. The cost for health life campaign could come from advertising in the press media, electronic media, or even from direct visit in school or hospital. Similarly, the cost for treatment intervention comes from cost for drug or any cost associated with treating the patients to get recovery as soon as possible. 


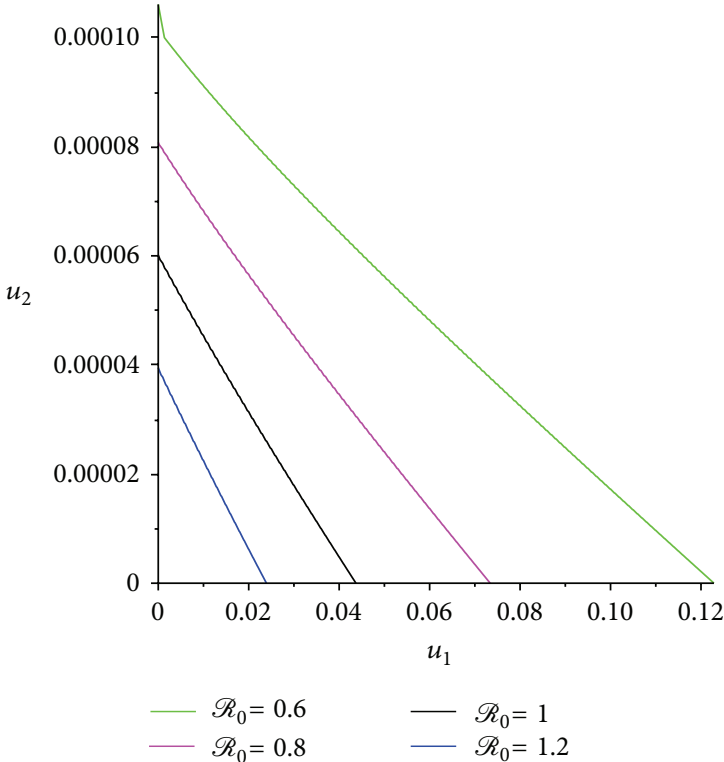

(a)

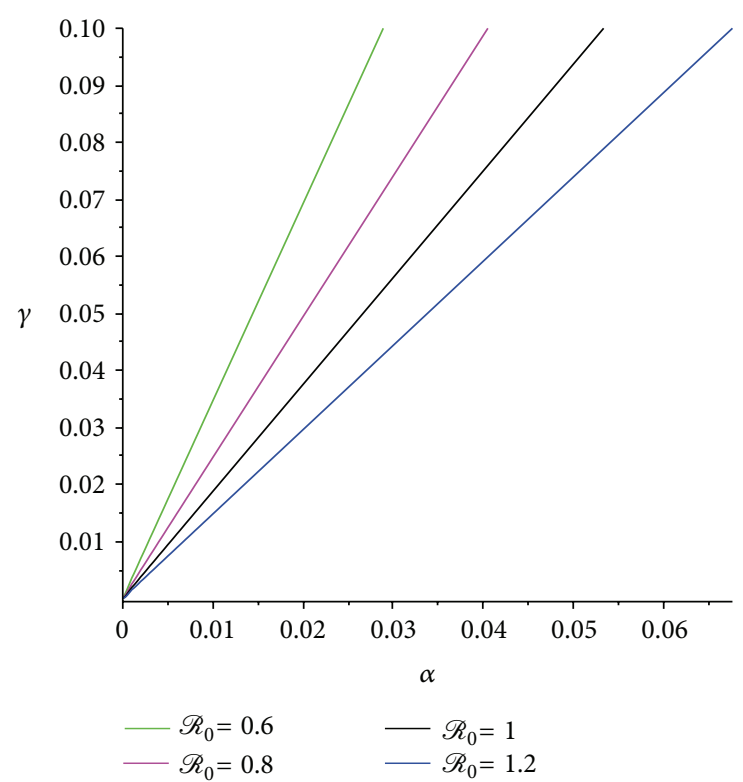

(b)

FIgURE 2: Parameter sensitivity of $\mathscr{R}_{0}$ between $u_{1}$ versus $u_{2}$ (a) and $\alpha$ versus $\gamma$ (b).

We would like to find the value of control variable $\widehat{u}_{1}(t)$ and $\widehat{u}_{2}(t)$ from $t=0$ to $t=T$ such that

$$
J\left(\widehat{u}_{1}(t), \widehat{u}_{2}(t)\right)=\min \left\{J\left(u_{i}^{*}, \Omega\right) \mid\left(u_{1}, u_{2}\right) \in \Phi\right\},
$$

subject to system (1) and where the control set $\Phi$ is a piecewise function on $[0, T]$ and $a_{i} \leq u_{i}^{*}(t) \leq b_{i}$ for $i=1,2$. Tehese parameters, $a_{i}$ and $b_{i}$, are the lower and upper bounds of the control variables, respectively. The existence of optimal controls $\left(u_{i}^{*}\right), i=1,2$, comes from convexity of the integrand of (9) with respect to controls and regularity of the system (1).

To find the optimal control for $u_{1}$ and $u_{2}$ in order to minimize the cost function in (9), we begin with defining a Lagrangian for the systems (1) and (9). The Lagrangian for the problem in here consists of the integrand of the objective functional and the inner product of the right hand sides of the state equations and the adjoint variables $\Lambda=\left(\lambda_{x}, \lambda_{y}, \lambda_{z}\right)$. We define our Lagrangian as given by

$$
\begin{aligned}
\mathscr{L}(\Omega, \Lambda)=\omega_{x} x^{2}+\omega_{y} y^{2}+\omega_{z} z^{2}+\omega_{u_{1}} u_{1}^{2}+\omega_{u_{2}} u_{2}^{2} \\
+\lambda_{x}\left(\theta(x+p y+q z)+\left(\gamma+u_{1}(t)\right) y\right. \\
-(b y+b z) x-\mu x) \\
+\lambda_{y}(\theta((1-p) y+(1-q) z) \\
+(b y+b z) x+u_{2}(t) z \\
\left.-\left(\gamma+u_{1}(t)\right) y-(b z+\alpha) y-\mu y\right) \\
+\lambda_{z}\left((b z+\alpha) y-u_{2}(t)-\mu z\right) .
\end{aligned}
$$

The adjoint equation variables $\Lambda=\left(\lambda_{x}, \lambda_{y}, \lambda_{z}\right)$ correspond to the system of state equation (1) that is taken from partial derivative of the Lagrangian function with respect to each state variables and is given by

$$
\begin{aligned}
\dot{\lambda}_{x}= & -\frac{\partial \mathscr{L}}{\partial x} \\
= & -2 \omega_{x} x+\lambda_{x}(\mu-\theta)+\left(\lambda_{x}-\lambda_{y}\right)(b y+b z), \\
\dot{\lambda}_{y}= & -\frac{\partial \mathscr{L}}{\partial y} \\
= & -2 \omega_{y} y-\lambda_{x} p y+\left(\lambda_{y}-\lambda_{1}\right)\left(\gamma+u_{1}(t)\right) \cdots \\
& +b x\left(\lambda_{x}-\lambda_{y}\right)+\left(\lambda_{y}-\lambda_{z}\right)(b z+\alpha) \\
& -\lambda_{y}(\theta(1-p)-\mu), \\
\dot{\lambda}_{z}= & -\frac{\partial \mathscr{L}}{\partial z} \\
= & -2 \omega_{z} z-\lambda_{1} q \theta+b x\left(\lambda_{x}-\lambda_{y}\right)-\lambda_{y}(1-q) \theta \ldots \\
& +b y\left(\lambda_{y}-\lambda_{z}\right)+u_{2}(t)\left(\lambda_{z}-\lambda_{y}\right)+\lambda_{z} \mu .
\end{aligned}
$$

The terminal condition of adjoint equation system (12) is equal to zero $\left(\lambda_{x}(T)=0, \lambda_{y}(T)=0\right.$, and $\left.\lambda_{z}(T)=0\right)$.

To obtain the optimality condition in order to minimize the cost function (9), we also will differentiate the lagrangian $\mathscr{L}$ with respect to each control variable and set it to equal to zero. Therefore, we have

$$
\begin{aligned}
& \frac{\partial \mathscr{L}}{\partial u_{1}}=2 \omega_{u_{1}} u_{1}+y\left(\lambda_{x}-\lambda_{y}\right)=0, \\
& \frac{\partial \mathscr{L}}{\partial u_{2}}=2 \omega_{u_{2}} u_{2}+z\left(\lambda_{y}-\lambda_{z}\right)=0 .
\end{aligned}
$$




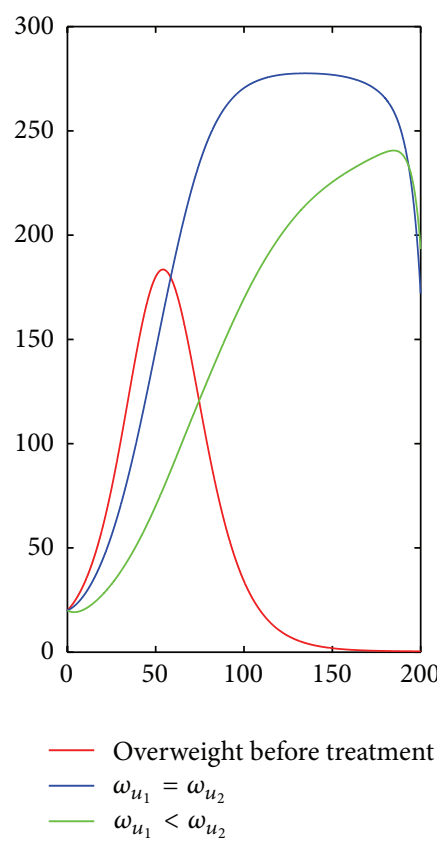

(a)

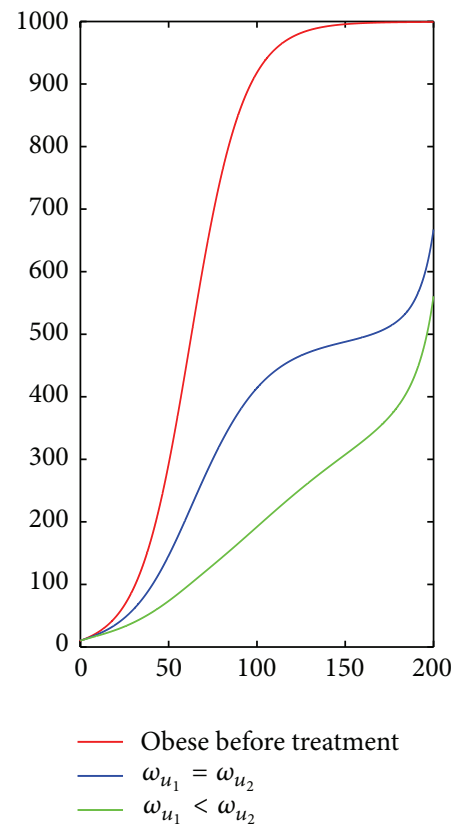

(b)

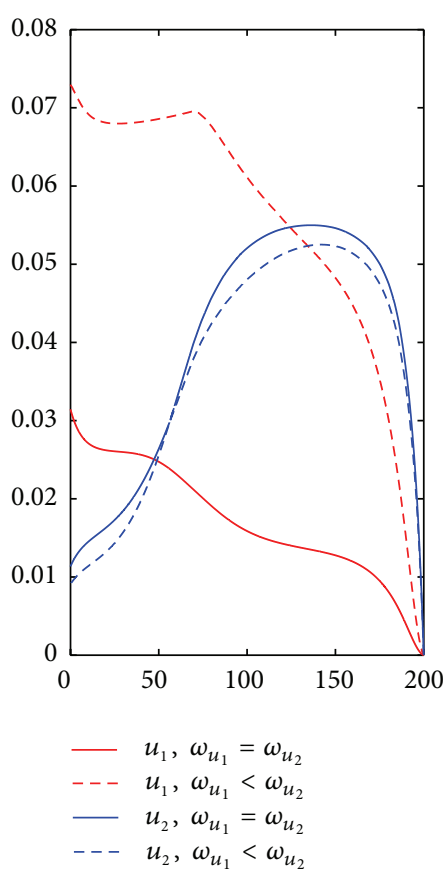

(c)

FIGURE 3: Numerical simulations of overweight (a) and obese (b) compartments and control variable (c) with different conditions of $\omega_{u_{1}}$ and $\omega_{u_{2}}$.

Solving (13), we obtain that

$$
\widehat{u}_{1}=\frac{y\left(\lambda_{y}-\lambda_{x}\right)}{2 \omega_{u_{1}}}, \quad \widehat{u}_{2}=\frac{z\left(\lambda_{z}-\lambda_{y}\right)}{2 \omega_{u_{2}}} \text {. }
$$

Consider the lower bound $a_{i}$ and upper bound $b_{i}$ for $i=1,2$ withrespect to each control variable, the optimal control now is characterized as

$$
\begin{aligned}
& \widehat{u}_{1}=\max \left(a_{1}, \min \left(b_{1}, \frac{y\left(\lambda_{y}-\lambda_{x}\right)}{2 \omega_{u_{1}}}\right)\right), \\
& \widehat{u}_{2}=\max \left(a_{2}, \min \left(b_{2}, \frac{z\left(\lambda_{z}-\lambda_{y}\right)}{2 \omega_{u_{2}}}\right)\right) .
\end{aligned}
$$

Now, we point out that our optimal control system consists of the state system (1) with initial condition given for $t=0$, the adjoint system (12) with final condition given for $t=T$ ( $T$ is a final time) and the optimality condition in (15). The optimal control problem will be solved with iterative method forward in time for state equation and backward in time for adjoint equation. The controls are updated in each iteration until the convergence condition is reached. Further application of optimal control theory in contagious disease could be seen in [12]. In the next section, some numerical simulation for optimal control problem will be shown for different cases which represent the possibility condition in the field.

\section{Numerical Simulation}

The dynamical model in this paper considered the obese spread via daily social interaction and the data for simulation is given in Table 1. In the following three subsections, we discuss the simulation results for different cases, that is, for different weight cost for each control variable, different initial condition for each compartments, and different value for some parameter in autonomous system (1).

5.1. Variation of Weight Cost for Control Variable. This condition describes the situation where the cost for application in the field is different between campaign and rehabilitation intervention. We balance the human populations and control functions in cost functional while choosing the weight cost such that $\omega_{u} / \omega_{x} \approx u^{2} / f^{2}$ with $f \in \Omega$ because the magnitudes of human populations and the control variables are on different scales.

5.2. Variation of the Initial Condition for the State Equation. Simulations in this subsection are given to accommodate the different initial condition for state equation, let us call it a prevention scenario where the number of obese people is relatively low $(x(0)=970, y(0)=20, z(0)=10)$ and endemic reduction scenario where number of obese people is relatively high $(x(0)=830, y(0)=20, z(0)=150)$. The simulation is given with same value for all parameters and also $\omega_{u_{1}}=\omega_{u_{2}}$.

The numerical results for this subsection are obtained for different values of $\omega_{u_{i}}$ while keeping the other parameters and initial conditions for state and adjoint variables the 


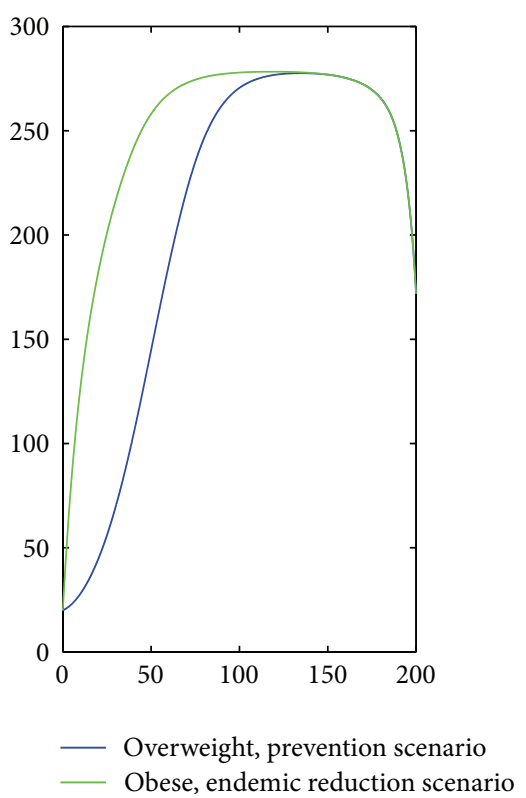

(a)

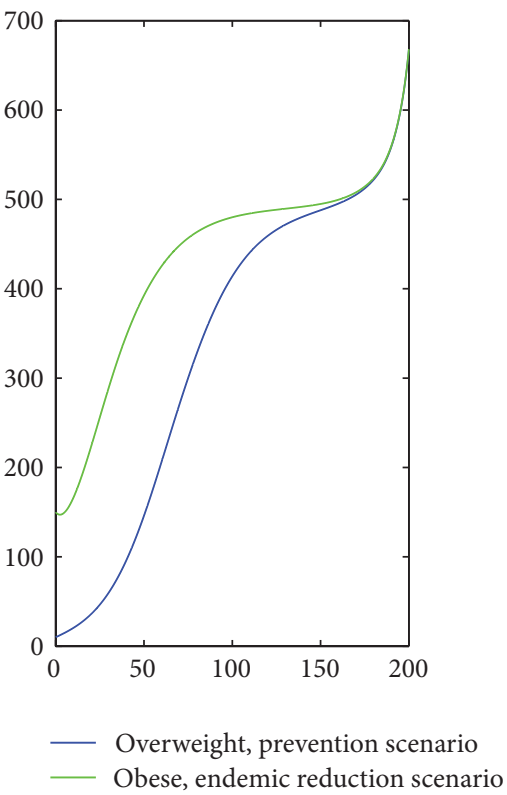

(b)

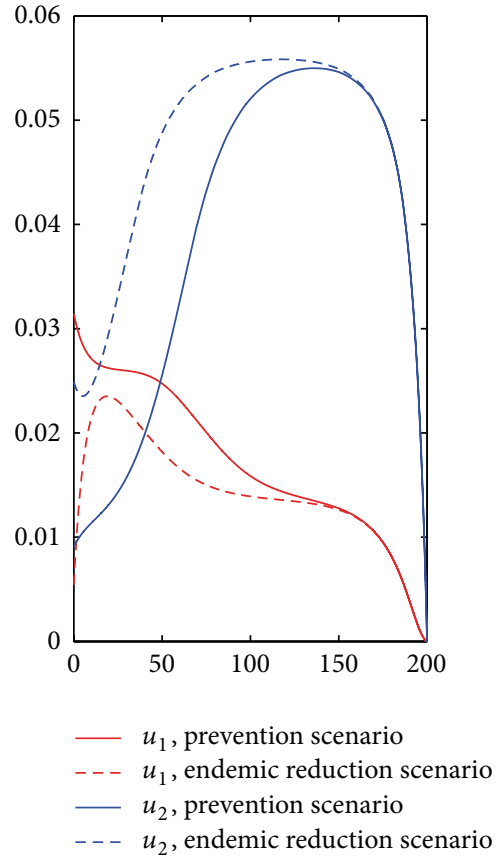

(c)

FIGURE 4: Numerical simulations of overweight (a) and obese (b) compartments and control variable (c) for prevention and endemic reduction scenario.

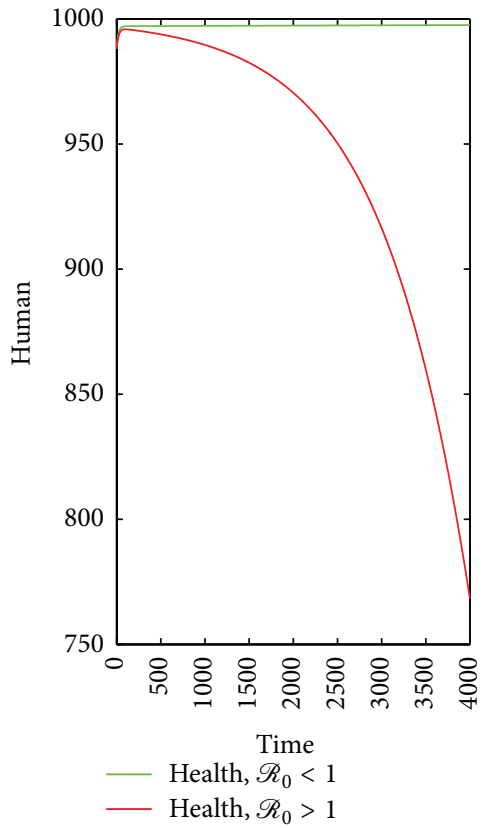

(a)

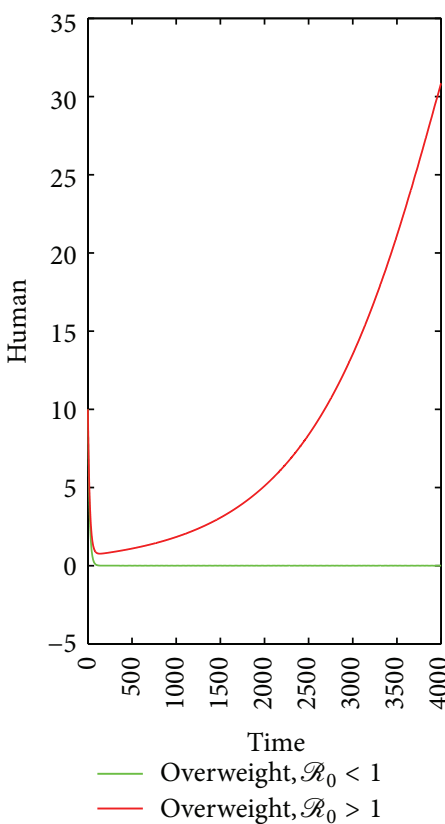

(b)

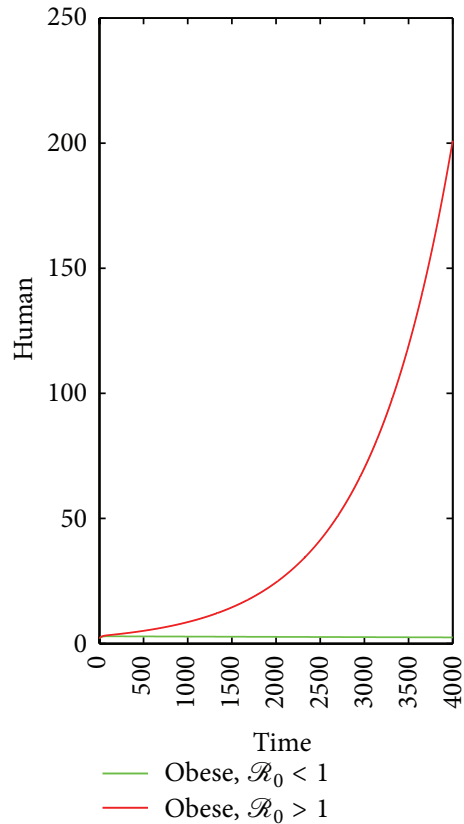

(c)

FIgURE 5: Numerical simulations of healthy (a), overweight (b), and obese (c) compartments when $\mathscr{R}_{0}<1$ and $\mathscr{R}_{0}>1$.

same. From Figure 3(b), it can be seen that intervention of treatment and health life campaign together success to minimize number of obese people. In the other hand, because number of obese people decrease, total number of overweight people is increasing because of success of the treatment for obese people (see Figure 3(a)).
This phenomenon appears because some people from obese compartment move to overweight compartment as a result of rehabilitation programme. From Figure 3(c), it is shown that if $\omega_{u_{1}}<\omega_{u_{2}}$, then the rate of $u_{1}$ will be much more larger than when $\omega_{u_{1}}=\omega_{u_{2}}$. As a result, with control rate depend on time like Figure 3(c), it can be seen that 
number of overweight and obese people is become smaller when $\omega_{u_{1}}<\omega_{u_{2}}$ rather than when $\omega_{u_{1}}=\omega_{u_{2}}$. This situation as a consequence when health life rate campaign $\left(u_{1}\right)$ become larger in a whole time. When weight cost for health life campaign rate become smaller, then the performance index of cost function become smaller, i.e from $388611\left(\omega_{u_{1}}=\omega_{u_{2}}\right)$ to $195520\left(\omega_{u_{1}}<\omega_{u_{2}}\right)$.

From Figure 4 , it can be seen that endemic reduction case needs a larger rate of $u_{1}$ and $u_{2}$ to reduce the number of obese people in the final time $(t=T)$, same as in the prevention scenario. As a result, the cost function becomes much more larger from 388611 (prevention scenario) to 514940 (endemic reduction scenario). Therefore, to make the programme of reducing number of obese incidence more success, it will be better to give the intervention of health life campaign and treatment programme at the beginning of endemic (prevention scenario) because the cost is much lower than if we give the interventions when endemic already happens. This is often overlooked by the policy makers in terms of mass action for obesity management process.

5.3. Variaton of Interaction Coefficient Parameters. We perform the last simulations in this subsection for the autonomous system (1) for case when $\mathscr{R}_{0}<1$ and $\mathscr{R}_{0}>1$. For case when $\mathscr{R}_{0}=1.58$, all parameters are the same with description in Table 1 , while for $\mathscr{R}_{0}=0.79$, the difference is only in the value of $b$ which is equal to $0.05 / 1000$. It can be seen in Figure 5 that if $\mathscr{R}_{0}<1$, then the dynamic of system (1) will tend to be disease-free equilibrium, while if $\mathscr{R}_{0}>1$, then the endemic goes to equilibrium where all compartments exist in the field. From this simulation, we can see that reducing social interaction between overweight and obese people (b) will reduce number of overweight and obese people. These results show that social interaction with overweight and obese people could increase number of new incidents of obesity. It has been suggested that changing this social interaction rate may lead to decline of obesity.

Other alternative way to reduce $\mathscr{R}_{0}$ could be choosen according to the form of $\mathscr{R}_{0}$ in (8), for example, maximizing portion of healthy recruitment rate $(p$ and $q)$, maximizing healthy life campaign $\left(u_{1}\right)$, maximizing recovery rate from obese compartment to healthy compartment $(\gamma)$, and so on.

\section{Conclusion}

Mathematical model for obesity in a closed population has been constructed in this paper. Vertical transmission from infectious parents has been accommodated into the model as well as the intervention program like healthy life campaign and treatment/rehabilitation program. Diseasefree equilibrium and endemic equilibrium have been shown analytically. Basic reproductive ratio as the endemic indicator has been obtained for local stability criteria of disease-free equilibrium.

With the optimal rate, it is shown that the intervention program with healthy life campaign and rehabilitation has significantly reduced number of obese people. The numerical results show that it is much better to start the program in the early situation where number of obese people is relatively low rather than to wait until the number of obese people is relatively high.

Further work should be done to include different social interaction rates for each compartment and different age classes as well as to accommodate the cross-marriage among the parents.

\section{Conflict of Interests}

The authors declare that there is no conflict of interests regarding the publication of this paper.

\section{Acknowledgments}

The authors thank the reviewer for all the constructive comments. This research is funded by the research grant of the Indonesian Directorate General for Higher Education.

\section{References}

[1] World Health Organization, "Obesity: preventing and managing the global epidemic," WHO Technical Report no. 894, WHO, Geneva, Switzerland, 2000, http://www.who.int/nutrition/.

[2] S. S. Deusinger, R. H. Deusinger, and S. B. Racette, “The obesity epidemic: health consequences and implications for physical therapy," Physical Therapy, vol. 12, no. 6, pp. 82-104, 2004.

[3] World Health Organization, "Obesity and overweight: media center: keys fact," http://www.who.int/mediacentre/.

[4] R. W. Taylor, K. A. McAuley, W. Barbezat, V. L. Farmer, S. M. Williams, and J. I. Mann, "Two-year follow-up of an obesity prevention initiative in children: the APPLE project," American Journal of Clinical Nutrition, vol. 88, no. 5, pp. 1371-1377, 2008.

[5] N. A. Christakis and J. H. Fowler, "The spread of obesity in a large social network over 32 years," The New England Journal of Medicine, vol. 357, no. 4, pp. 370-379, 2007.

[6] A. L. Hill, D. G. Rand, M. A. Nowak, and N. A. Christakis, "Infectious disease modeling of social contagion in networks," PLoS Computational Biology, vol. 6, no. 11, Article ID e1000968, 2010.

[7] W. C. Knowler, M. F. Saad, D. J. Pettitt, R. G. Nelson, and P. H. Bennett, "Determinants of diabetes mellitus in the Pima Indians," Diabetes Care, vol. 16, supplement 1, pp. 216-227, 1993.

[8] R. C. Whitaker, J. A. Wright, M. S. Pepe, K. D. Seidel, and W. H. Dietz, "Predicting obesity in young adulthood from childhood and parental obesity," The New England Journal of Medicine, vol. 337, no. 13, pp. 869-873, 1997.

[9] O. Diekmann, J. A. P. Heesterbeek, and M. G. Roberts, “The construction of next-generation matrices for compartmental epidemic models," Journal of the Royal Society Interface, vol. 7, no. 47, pp. 873-885, 2010.

[10] O. Diekmann and J. A. P. Heesterbeek, Mathematical Epidemiology of Infectious Diseases: Model building, Analysis and Interpretation, John Wiley \& Sons, Chichester, UK, 2000.

[11] A. Hurford, D. Cownden, and T. Day, "Next-generation tools for evolutionary invasion analyses," Journal of the Royal Society Interface, vol. 7, no. 45, pp. 561-571, 2010.

[12] D. Aldila, T. Götz, and E. Soewono, "An optimal control problem arising from a dengue disease transmission model," Mathematical Biosciences, vol. 242, no. 1, pp. 9-16, 2013. 


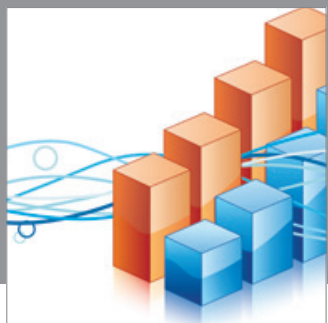

Advances in

Operations Research

mansans

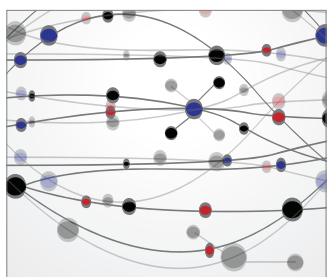

The Scientific World Journal
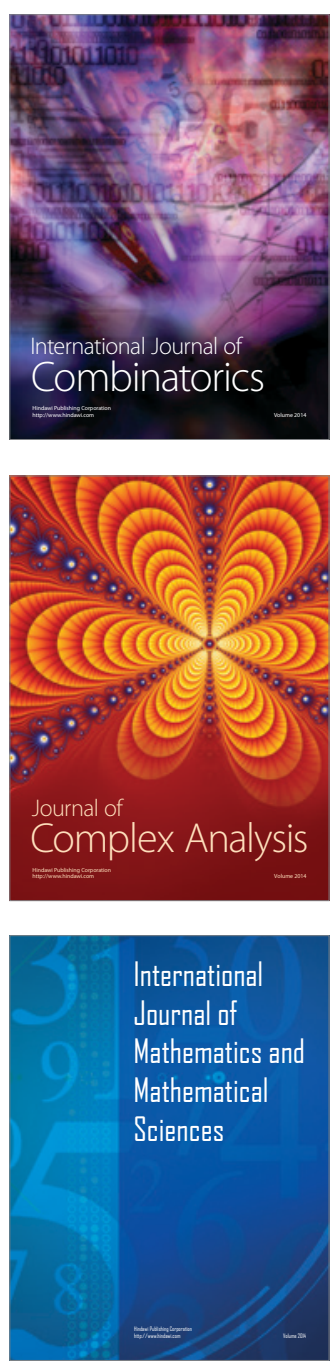
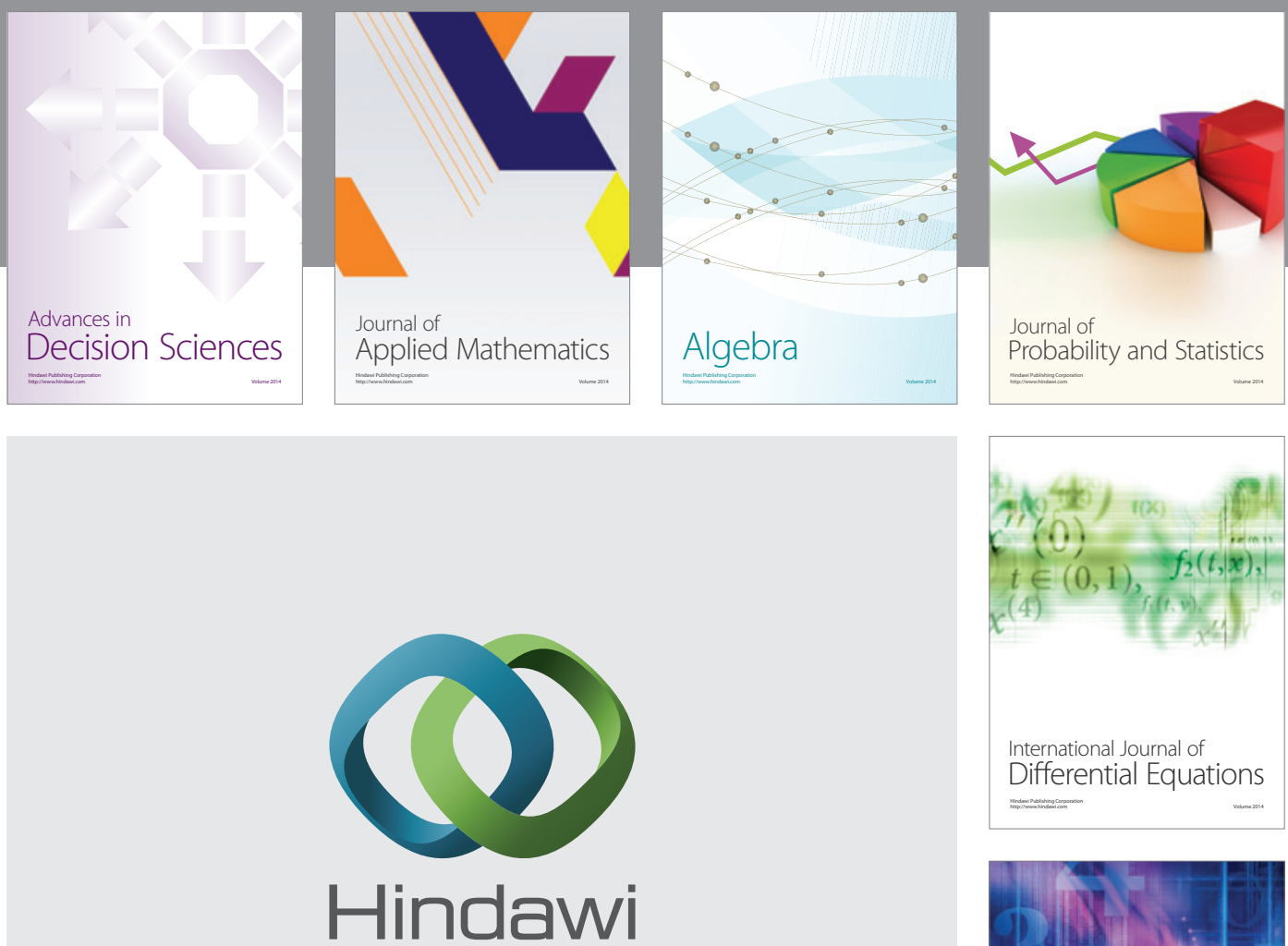

Submit your manuscripts at http://www.hindawi.com
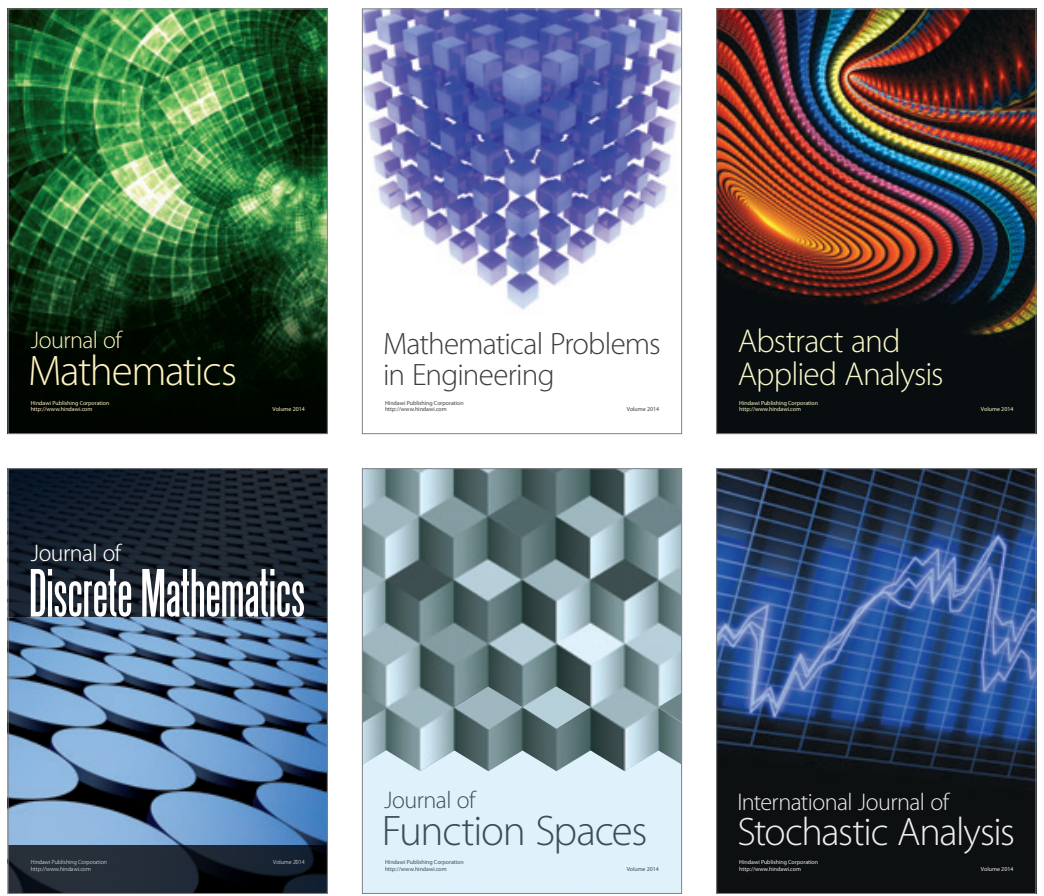

Journal of

Function Spaces

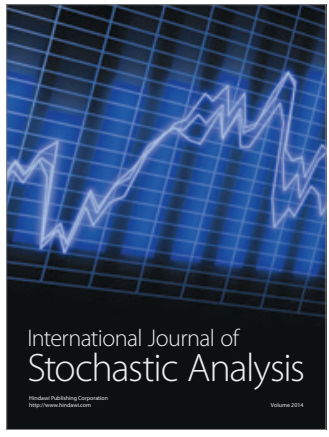

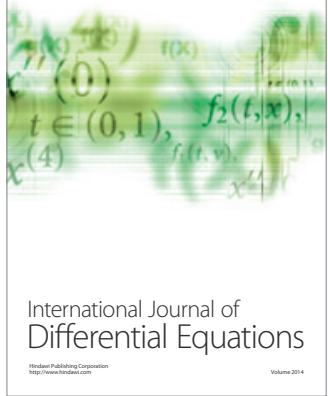
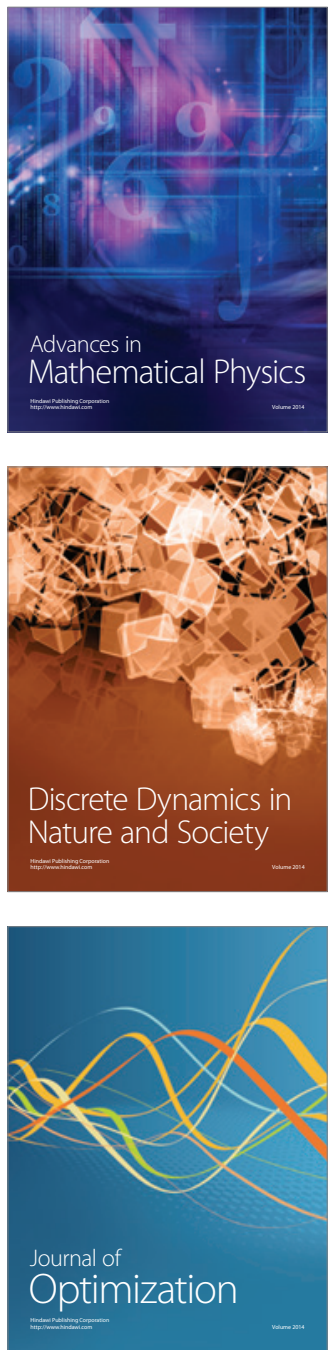